\title{
Dinámica sucesional del bosque luego de establecer fajas de enriquecimiento, El Caimital, Barinas - Venezuela
}

\author{
Forest's successional dynamics, after stablish enrichment strips, Caimital, Barinas -Venezuela
}

Historial del artículo
Recibido:
12 de febrero de 2021
Revisado
25 de mayo de 2021
Aceptado:
09 de junio de 2021

09 de junio de 2021

\author{
Marherir Vanesa Pino Suarez ${ }^{\mathrm{a}}$, Yeritsa Josefina Rojas Uzcategui ${ }^{\mathrm{b}}$, Pedro \\ Salcedo $^{\mathrm{c}}$, Ronald Edgardo Rangel Vasquez ${ }^{\mathrm{d}}$, Alicia Elena Gómez Mejías ${ }^{\mathrm{e}}$ \\ a Universidad de Los Andes - ULA, Mérida, Venezuela \\ ORCID: https://orcid.org/0000-0002-2883-5902 \\ ${ }^{\mathrm{b}}$ Universidad de Los Andes - ULA, Mérida, Venezuela \\ ORCID: https://orcid.org/0000-0001-5638-7126
}

c Universidad de Los Andes - ULA, Mérida, Venezuela ORCID: https://orcid.org/0000-0002-7838-9234

\section{Palabras clave}

Composición florística, fisiografía de banco, sucesión, Venezuela.

\section{Keywords}

Banco physiography, floristic composition, succession, Venezuela

\begin{abstract}
Resumen
Se determinó la composición florística, estructura y dinámica, luego de 57 años de haber sido establecidas fajas de enriquecimiento en un sector del Bosque Universitario "El Caimital”, municipio Obispo, estado Barinas, Venezuela. Se seleccionaron dos áreas: una conformada por el bosque primario (testigo) y otra por un lote del bosque donde se establecieron las fajas de enriquecimiento. En campo se utilizaron: a) tres parcelas de 1,3 ha para evaluar el lote completo donde se encuentra ubicado el sistema silvicultural; b) tres parcelas de 1 ha para el levantamiento de las testigos seleccionadas al azar, donde fueron evaluados los individuos mayores a $10 \mathrm{~cm}$ de diámetro a la altura de pecho (DAP), en cada una de ellas se establecieron tres sub-parcelas de $20 \mathrm{~m}^{2}$ para estudiar todas las especies presentes en el sotobosque. El sector del bosque estudiado está ubicado sobre la unidad fisiográfica de banco. En el bosque no intervenido (BNI), se encontraron 49 especies arbóreas agrupadas en 24 familias y en el bosque intervenido (BI) se encontraron 45 especies en 23 familias, siendo las familias Arecaceae, Leguminosae, Moracea, Malvaceae y Euphorbiaceae las más representativas para ambos tipos de bosques. Según el Índice de Valor de Importancia (IVI) e Índice de Importancia Ampliado (IIA), las especies más importantes del (BNI): Trophis racemosa, Attalea butyraceae y Lonchocarpus pictus. Para el (BI) son las especies Attalea butyracea, Hura crepitans y Lonchocarpus pictus. El área de estudio presentó alta diversidad con un Índice de Margalef mayor a 5,0.
\end{abstract}

\begin{abstract}
The floristic composition, structure and dynamism were determined after 57 years of enrichment strips, located in a University Forest EL Caimital, municipio Obispo, Barinas - Venezuela. Two areas were selected: one formed by the primary forest (witness) and another one formed by Fajas de Enriquecimiento in a secondary forest. In the forest were evaluated 3 plots of 1,3 ha (enrichment strips) and 3 plots of 1 ha random selected in the primary forest (witness). Were evaluated individuals with diameters bigger than $10 \mathrm{~cm}$, in each one of that plots, three sub-plots of $20 \mathrm{~m}^{2}$ were measured, to study all the species in the understory. The forest is in the Banco's fisiography. In the primary forest, 49 tree species grouped in 24 families were found and 45 species grouped in 23 families in the secondary forest. The most representative families were Arecaceae, Leguminosae, Moracea, Malvaceae and Euphorbiaceae to both forests. In the Importance Value Index (IVI) and Expanded Importance Index (IIA) the most representative species were Trophis racemosa, Attalea butyraceae y Lonchocarpus pictus (primary forest) and Attalea butyracea, Hura crepitans y Lonchocarpus pictus in (secondary forest). The Margalef's index
\end{abstract} were $>5$. 


\section{Introducción}

El Bosque Universitario "El Caimital" (BUEC) constituye el único relicto boscoso que queda en las cercanías de la población del mismo nombre en el municipio Obispos del estado Barinas y donde se han realizado importantes estudios silviculturales que podrían ser de interés para su implementación a mayor escala. Gran parte de las investigaciones se han centrado hacia los diferentes métodos de recuperar o mantener la superficie forestal, así como incrementar el valor económico del bosque mediante plantaciones con especies nativas y exóticas, a su vez reduciendo las perturbaciones inherentes a otras técnicas a campo abierto y resguardando la diversidad biológica, minimizando los espacios sometidos a explotación intensiva.

El sistema silvicultural de fajas de enriquecimiento o enriquecimiento consiste en el aumento de especies de alto valor económico sobre la estructura del bosque (Lamprecht, 1990; Zulle et al., 2015). En general el procedimiento consiste en la apertura mecanizada o manual de fajas dentro del bosque remanente (aprovechado), cortando toda la vegetación en un ancho de unos 3-5 metros y longitud variable, para allí plantar los individuos de especies valiosas (Lirio et al., 2010; Lozada et al., 2003). Además, este tipo de sistema tiene un efecto en el desarrollo de las plantas, producto de factores ecológicos que se relacionan con el tipo y grado de protección lateral que ofrece la vegetación remanente entre las fajas o interfajas (Alvarez \& Lara, 2008). Este sistema es muy utilizado en especies valiosas que tienen dificultades para su regeneración natural.

Dentro de las experiencias de esta técnica silvicultural podemos encontrar: a) Zulle et al. (2015) encontraron en un bosque explotado del Chaco húmedo en Argentina que, luego de seis años de la plantación, solo Peltophorum dubium obtuvo buenos resultados de sobrevivencia y que el crecimiento (diámetro y altura) fue pobre debido a la alta competencia. b) Lirio et al. (2010) reportan para Venezuela que las tasas de crecimiento para la Guayana Venezolana bajo este sistema han sido muy bajas, sin embargo, se han observado que los individuos ubicados al lado de las carreteras han alcanzado mejores desarrollos. c) Flores et al. (2004) presentaron resultados de la especie Cedrelinga catenaeformis en Perú, con el mejor crecimiento y productividad tanto en fajas de enriquecimiento como en plantaciones puras. d) Álvarez \& Lara (2008) concluyeron que las plantaciones de Nothofagus dombeyi en fajas resultaron adecuadas para su desarrollo inicial con objetivos de restauración ecológica, en bosques del tipo CoigueRaulí-Tepa (Chile) degradados por tala con fines madereros.
En 1963 se implementó el sistema silvicultural "fajas de enriquecimiento", dentro del (BUEC), el cual constó de 15 fajas equidistantes de longitud variable y acondicionada; esto con el fin de llevarlas a una producción continua por tiempo indefinido (Benítez et al., 1987). Sin embargo, la ausencia de monitoreo continuo a partir de su establecimiento dificulta el diagnóstico y la evaluación de patrones de desarrollos y por consiguiente los comportamientos particulares en el transcurso de crecimiento de diferentes especies arbóreas.

En Venezuela se han realizado algunas investigaciones sobre las "fajas de enriquecimiento" implementadas en las masas boscosas dentro de los cuales se encuentran Kammesheidt et al. (2001), Lozada et al. (2003) y Lozada et al. (2011), aun así, existen pocos estudios en el país que nos puedan demostrar la eficiencia del método silvicultural nombrado anteriormente.

Para las fajas de enriquecimiento establecidas en El Caimital, Vincent (1969), menciona los buenos resultados preliminares con Cordia thaisiana plantado por stump (tocón) en bosque sin envenenamiento o poco clareado. Se ha logrado una altura de alrededor de 3 metros en tres años. En algunas fajas se ha logrado un crecimiento de 2 a 2,5 en 3 o 4 años con Swietenia macrophylla.

Benítez et al. (1987) afirman que, para el establecimiento de las 15 fajas equidistantes, se eliminó la vegetación natural en fajas de un ancho de 2 metros y se plantaron mediante stump y siembra directa un número variable de Tabebuia rosea, Sterculia apetala, Swietenia macrophylla, Ceiba pentandra, Hura crepitans, Cordia thaisiana y Albizia saman. Desde entonces a la fecha se han efectuado replantes en algunas de las fajas, cuyo número, intensidad, especies y dimensiones no fue posible conocer.

La ausencia de información completa, principalmente del intervalo entre la plantación inicial y la evaluación actual, impide realizar un diagnóstico de supervivencia. La especie Albizia saman, presente en 9 fajas con 55 individuos, y Hura crepitans, en 11 fajas con 798 individuos, indica la buena adaptabilidad de estas a las condiciones del bosque considerado. Aun cuando hubiera habido replantes en el intervalo de tiempo considerado, después de 24 años del establecimiento de las fajas, 1.982 individuos de los 1.687 censados, se encuentra en las dos primeras categorías: 0-10 y 10-20 cm DAP (diámetro de altura de pecho). La especie Hura crepitans posee el mayor número de individuos (47.3\%), seguido por Tabebuia rosea $(40,8$ $\%$, Benítez et al., 1987). 


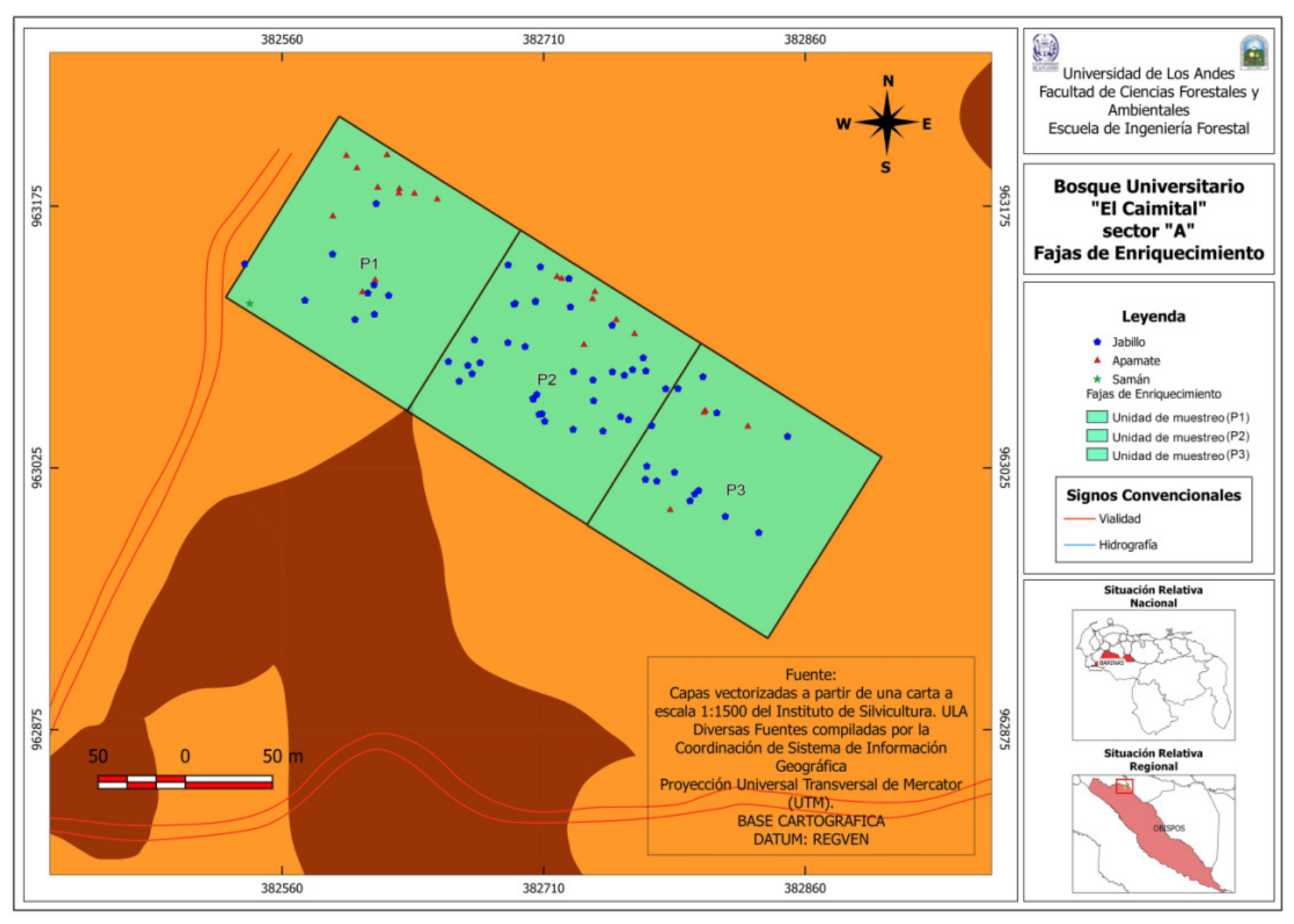

Figura 1. Ubicación relativa nacional, regional y local del área de estudio. Fuente: Elaboración propia.

Del mismo modo Lozada et al. (2003) explican que a los 24 años de haberse establecido el sistema en El Caimital se obtuvieron los siguientes resultados en el crecimiento (diámetro, en cm/año): Tabebuia rosea $(0,57)$, Swietenia macrophylla $(0,38)$, Hura crepitans $(0,63)$, Cordia alliodora $(0,22)$, y Pithecellobium saman $(1,18)$.

Para alcanzar un buen estudio de la dinámica sucesional de un bosque se hace necesario contar con un monitoreo que permita analizar los cambios que ocurren en el tiempo (mortalidad, reclutamiento) de las especies. En la actualidad el BUEC es un paisaje que se ve amenazado por la extensión urbana en todo su entorno, es de gran importancia su monitoreo para conocer así sus dinámicas y generar diferentes parámetros y lineamientos en pro de la conservación de su biodiversidad.

Debido a la escasez de información, el propósito de este estudio fue analizar la composición florística, estructura y dinamismo del Bosque Universitario El Caimital (BUEC), con miras de clarificar el avance de la sucesión luego del establecimiento de las fajas de enriquecimiento, realizado en 1963, así como las evaluaciones posteriores realizadas por Vincent (1969), Benítez et al. (1987), Lozada et al., (2003) y la del presente estudio.

\section{Materiales y métodos}

\section{Metodología de campo}

El bosque "Caimital" también llamado "Bosque del Río Yuca", debido a su extensión hasta las orillas de este y a las cercanías de su confluencia con el Masparro; está situado en la región Noreste del estado Barinas (figura 1), específicamente en el Distrito Obispos. Limita por el oeste con la parte vieja del camino de Barrancas, Caimital y Obispos, por el norte con el río Yuca, siendo por el sur y el este límites con terrenos pertenecientes al Instituto Agrario Nacional (IGN) presentando una altitud media sobre el nivel del mar de 200 metros aproximadamente (Montilla \& Rivas, 1987; Moret et al., 2010). 
La superficie aproximada es de unas 800 hectáreas, con una topografía por lo general plana y condiciones fisiográficas de banco, sub-banco y bajío de los bosques llaneros. La pendiente no excede al 3 \% (Moret et al., 2010). Los suelos son de origen aluvial y aluvio-lacustrino, formados por materiales arrastrados por las aguas desde las formaciones montañosas vecinas. En detalle pueden distinguirse dos series: río Yuca I (pH 5,5 a 6) y río Yuca II (pH 5,1), con características bien definidas (Bernal, 1967). Los suelos corresponden a Regosoles aluviales con procesos de ferretización y que tienden, como clímax, a la laterita (Moret et al., 2010).

Climáticamente, se pueden observar dos épocas bien marcadas, el invierno, de mayo a noviembre $(220 \mathrm{~mm})$ y el verano desde diciembre hasta abril. Las precipitaciones anuales promedio oscilan en 1.500 a $2.200 \mathrm{~mm}$ por año. La temperatura media anual del bosque "Caimital", se estima entre 26,0 y $26,5^{\circ} \mathrm{C}$. La humedad relativa anual media tiene valores próximos al $80 \%$ (Montilla \& Rivas, 1987).

Según Montilla \& Rivas (1987), atendiendo a la clasificación de Pittier, el bosque "Caimital" pertenece a la formación tropófito macrotérmico; se pueden diferenciar tres grupos ecológicos: bosque de galería, encontrándose en las proximidades del río Yuca debido a la altura de la capa freática; bosque tropófico y transición. En la clasificación de Holdrige, se sitúa como Bosque Tropical Seco. Es un bosque mixto e irregular.

Según datos recolectados del inventario hecho por Konrad (1961), comprende aproximadamente 68 especies de (DAP) mayor que $20 \mathrm{~cm}$ y sobre superficies reducidas se encontraron individuos de edades muy diferentes. Por otro lado, Bernal (1967) señala que se encontraron más de 80 especies en el estrato arbóreo. El coeficiente de mezcla posee valores comprendidos entre 1/5 y 1/7.

La composición de mezcla varia de un lugar a otro, siendo el bosque un mosaico compuesto por unidades florísticamente distintas, aunque cabe resaltar que existe un grupo de especies constantes que medran en todas las combinaciones florísticas existentes; unas de estas especies son Trophis racemosa, Attalea butyracea, Inga sapindoides, Pochota fendleri, Fissicalyx fendleri; siendo Pochota fendleri una de las especies que menos abundan en todo el bosque (Montilla \& Rivas, 1987).

Según Konrad (1961), se distinguen tres estratos en la estructura vertical: sotobosque inferior, medio y superior, debido a que las capas frecuentemente ocupan posiciones intermedias, especialmente en el caso del estrato inferior, cuya separación del sotobosque y estrato medio es particularmente difícil y la distinción no es fácil.

Desde el punto de vista sociológico, este es un bosque aparentemente secundario antiguo, encontrándose diferentes especies forestales como: Swietenia macrophylla, Cedrela odorata, Anacardium excelsum, Astronium graveolens, Pachira quinata, entre otras; en algunos sectores del bosque existen plantaciones de Gmelina arbórea, Tectona grandis, Cedrela odorata y Pachira quinata (Moret et al., 2010).

\section{Selección del área}

Se seleccionó un lote de 4 hectáreas donde fue establecido el sistema "fajas de enriquecimiento" (bosque intervenido, BI) en el año 1963, dentro del Bosque Universitario "El Caimital" en el Sector (A), sobre la fisiografía de banco. Para poder establecer comparaciones se seleccionaron 3 parcelas (1 ha c/u, bosque no intervenido, BNI) seleccionadas al azar bajo la fisiografía de banco.

\section{Diseño y levantamiento de las unidades de muestreo}

Para establecer comparaciones en cada caso se establecieron tres unidades de muestreo, en el ensayo (fajas de enriquecimiento - bosque intervenido, $\mathrm{BI}$ ) con dimensiones rectangulares de 100 metros x 130 metros $(1,3 \mathrm{ha} \mathrm{c} / \mathrm{u})$ con orientación este-oeste, divididas cada una en 12 cuadrículas de 33 metros x 33 metros aproximadamente. Para las unidades de muestreo testigo (bosque no intervenido, BNI), la dimensión fue 100 metros x 100 metros ( 1 ha c/u) con orientación Noreste (fisiografía de banco) divididas en 16 cuadrículas de 25 metros x 25 metros; dejando una zona buffer para ambos casos de 25 metros (efecto de borde).

En las unidades de muestreo levantadas se realizó un censo de los árboles con un DAP $\geq 10 \mathrm{~cm}$. Para estudiar todas las especies de espermatofitas inferiores a $10 \mathrm{~cm}$ de (DAP), se establecieron 3 subunidades de muestreo de 2 metros x 10 metros $\left(20 \mathrm{~m}^{2}\right)$.

\section{Colecta de la información}

Para el estrato superior se registraron todos los individuos con un DAP $\geq 10 \mathrm{~cm}$ (Vincent et al., 2000), se tomaron en cuenta parámetros cualitativos y cuantitativos, tales como la respectiva identificación de la especie, parcela, subparcela, diámetro a la altura de pecho (DAP en $\mathrm{cm}$ ), altura (total, fuste y copa en metros). Para registrar las especies de sotobosque (espermatofitas) inferiores a $10 \mathrm{~cm}$ de (DAP) 
se consideraron los siguientes aspectos (Corredor, 2001): identificación de las especies, abundancia en categorías por tamaño (0-1 metros; 1-3 metros y $>3$ metros).

\section{Metodología de gabinete}

La información obtenida en campo fue procesada para obtener variables de interés ecológico, se realizaron cálculos en Excel (2010) para el Índice de Valor Familiar (IVF), Índice de Valor de Importancia (IVI), Índice de Importancia Ampliado (IIA), curva especie-área, distribución por clases diamétricas y el Índice de Similaridad Beta Sorensen; esto fue procesado, por especie, parcela, tanto para el bosque intervenido (BI), como para el bosque no intervenido (BNI). La metodología para el cálculo de dichos índices aparece bien descrita en: Mori et al. (1983), Moret et al. (2010), Curtis \& McIntosh (1951), Lozada et al. (2011), Lozada (2010), Corredor (2001) y Zarco-Espinosa et al. (2010).

También se calcularon con el programa PAST3 versión 1.0.0.0, para el estrato superior y sostobosque, los Índices de Diversidad Alfa: a) riqueza específica (Riqueza, Margalef, Fisher). b) Estructura (Shannon y Simpson). c) Análisis de conglomerados (clúster), aplicado sobre las parcelas por tratamiento (bosque no intervenido-BNI, bosque intervenido-BI y bosque no intervenido-BNI versus bosque intervenido-BI) para ello se seleccionó el dendrograma de coeficiente de similitud Bray-Curtis para las comunidades potenciales.

\section{Cálculo de índices estructurales}

El Índice de Valor Familiar (IVF, Mori et al., 1983) permite observar el éxito ecológico de una familia en el área de estudio muestreada (Bustamante et al., 2009).

$\mathrm{IVFi}=\Sigma \% \mathrm{DvRFi}+\% \mathrm{DRFi}+\% \mathrm{DoRF}$

Donde:

IVFi = Índice de Valor Familiar: DvRFi = (número de especies de la familia i/ número de especies totales) x 100; $\mathrm{DRFi}=$ (número de individuos de la familia i/ número de individuos totales) $\mathrm{x} 100 ;$ DoRFi $=($ Área basal de la familia i/ Área basal total) x 100

El Índice de Valor de Importancia (IVI, Curtis \& McIntosh, 1951) se calcula para cada especie a partir de la suma de abundancia relativa, la frecuencia y la dominancia relativas. Permite comparar el peso ecológico de cada especie dentro del bosque (Alvis, 2009). Se expresa en términos porcentuales en una escala de 0 a 100 (Mora et al., 2013). Es un índice sintético estructural, cuyo objetivo es jerarquizar la dominancia de cada especie en la unidad de muestreo.

$\mathrm{IVI} \%=\mathrm{Ai} \%+\mathrm{Fi} \%+\mathrm{Di} \%$

Donde:

$\mathrm{Ai} \%=(\mathrm{Ai} / \mathrm{At}) \times 100$

$\mathrm{Ai} \%=$ es la relación porcentual del número de individuos de la especie con respecto al total de individuos de la unidad de muestreo; $\mathrm{Ai}=$ corresponde al número de individuos de una especie en una unidad de muestreo; At $=$ es el número total de individuos de todas las especies dentro de una unidad de muestreo.

$\mathrm{Fi}=(\mathrm{Ni} / \mathrm{N}) \times 100 ; \mathrm{Fi} \%=(\mathrm{Fi} / \mathrm{Ft}) \times 100$

$\mathrm{Fi}$ = es la relación porcentual entre el número de subunidades de muestreos en que aparece una especie $(\mathrm{Ni})$ y el total de sub-unidades de muestreo $(\mathrm{N}) ; \mathrm{Fi} \%=$ es la relación porcentual entre la frecuencia de una especie (Fi) y la suma de frecuencia de todas las especies (Ft).

$\mathrm{Di}=\Sigma \mathrm{ABi} ; \mathrm{Di} \%=(\mathrm{Di} / \mathrm{Dt}) \times 100$

$\mathrm{Di}$ = es la suma del área basal de todos los individuos de una especie; $\mathrm{Di} \%$ = es la relación porcentual entre la dominancia absoluta (Di) de una especie con respecto al área basal total de la unidad de muestreo (Dt). El área basal total es una medida indirecta de la biomasa y de la productividad de un sitio, se expresa en $\mathrm{m}^{2} / \mathrm{ha}$.

El área basal es la superficie de una sección transversal del tronco del individuo a la altura de pecho (1,30 metros de altura). Es un parámetro muy preciso. No hay solapamiento. Generalmente se toma la circunferencia a la altura de pecho (CAP en milímetros); excepcionalmente en diámetro a la altura de pecho (DAP en centímetros). Se calcula de la siguiente manera:

$\mathrm{AB}=(\Pi / 4) \times(\mathrm{DAP})^{2} ; \mathrm{AB}=(\mathrm{CAP})^{2} /(4 \Pi)$

El Índice de Importancia Ampliado (IIA, Lozada et al., 2011) incluye todas las formas de vida del sotobosque, cuyo tallo no llega a $10 \mathrm{~cm}$ de diámetro a la altura de pecho (DAP)

$\mathrm{IIA} \%=\mathrm{Ai} \%+\mathrm{Fi} \%+\mathrm{Di} \%+\mathrm{As} \%+\mathrm{Fs} \%$ 
Donde:

$\mathrm{As} \%=(\mathrm{As} \times 100) / \mathrm{Ast}$

$\mathrm{As} \%=$ abundancia porcentual de sotobosque; As = número total de individuos de una especie, encontrados en las sub-unidades de muestreo de sostobosque (DAP $<10$ $\mathrm{cm}) ;$ Ast $=$ número de individuos de todas las especies, encontrados en las unidades de muestreo de sotobosque.

$\mathrm{Fs} \%=(\mathrm{Fs} \times 100) / \mathrm{Fst}$

$\mathrm{Fs}=(\mathrm{Nis} / \mathrm{Ns}) \times 100$

$\mathrm{Fs} \%$ = frecuencia porcentual de sotobosque; Nis: número de unidades de muestreo de sostobosque en que aparece una especie; Ns: número de unidades de muestreo de sotobosque; Fst: es la suma de frecuencia de sotobosque de todas las especies.

Índice de Similaridad Sorensen (IS, Stiling, 1999). Los datos utilizados en este índice son de tipo cualitativos, de todos los coeficientes con datos cualitativos, el índice de Sorensen es el más satisfactorio. Este índice se basa en la relación presencia-ausencia de especies entre dos sistemas.

$\mathrm{S}=(2 \mathrm{c} /(\mathrm{a}+\mathrm{b})) \times 100$

Donde:

a: número total de especies en la comunidad $\mathrm{A}$; b: número total de especies en la comunidad B; c: número de especies comunes en ambas comunidades. Este índice presenta valores que van de 0 a 1 , significando la menor o mayor semejanza respectivamente, donde el 1 (100\%) indica que todas las especies se comparten.

El Índice de Margalef (Margalef, 1977) transforma el número de especies por muestra a una proporción a la cual las especies son añadidas por expansión de la muestra. Supone que hay una relación funcional entre el número de especies y el número total de individuos. Tiene en cuenta únicamente la riqueza de especies, pero de una forma que no aumente al aumentar el tamaño de la muestra (Valdez et al., 2018)

$\mathrm{D} \alpha=(\mathrm{S}-1) / \log \mathrm{N}$

Donde:

$\mathrm{S}=$ número de especies; $\mathrm{N}=$ número total de individuos. $\mathrm{A}$ mayor valor del índice será mayor la riqueza de especies.
Índice de $\alpha$ de Fisher (Fisher et al., 1943) sólo considera el número de especies $(\mathrm{S})$ y el total de individuos $(\mathrm{N})$ en las unidades de muestreo, además no depende del tamaño del área muestreada.

$\mathrm{S}=\alpha(\log \mathrm{e})\left(1+\frac{N}{\alpha}\right)$

Donde:

$\mathrm{S}=$ número de especies registradas en la muestra; $\mathrm{N}=$ total de individuos en la muestra; $\alpha=$ índice de diversidad.

Índice de Shannon-Wiener (H', Magurran, 1988) expresa la uniformidad de los valores de importancia a través de todas las especies de la muestra (Cabrera et al., 2018; Valdez et al., 2018). Este índice mide el grado promedio de incertidumbre para predecir la especie a la que pertenece un individuo tomado al azar dentro de las unidades de muestreo.

$$
\mathrm{H}^{\prime}=-\sum_{i=1}^{S} P i \ln (P i)
$$

Donde:

$\mathrm{S}=$ número de especies; $\mathrm{Pi}=$ proporción de individuos de la especie i. A mayor valor de H' mayor diversidad de especies. Este índice varía de 0 a log base 2 del número de morfoespecies censadas, determinadas por el número de morfoespecies presentes en la comunidad y basándose en la escala logarítmica escogida (Iannacone et al., 2003)

Índice de Dominancia Simpson $\left(\mathrm{D}_{\mathrm{Si}}\right.$, Campo \& Duval, 2014), también conocido como índice de dominancia y permite evaluar cuál es la especie que se encuentra en mayor proporción en un muestreo (Salazar et al., 2019). Considera la probabilidad que dos individuos de la población tomados al azar sean de la misma especie. Indica la relación existente entre riqueza y número de especies y la abundancia o número de individuos por especies.

$$
\mathrm{D}_{\mathrm{Si}}=\sum_{I=1}^{S} P i^{2}
$$

Donde:

pi: igual a la proporción entre ni y $N$; ni: número de individuos de la especie $i$.

El Índice de diversidad Riqueza (McIntosh, 1967) se refiere a la abundancia de especies por individuo; es decir el número de especies dividido por el número de individuos muestreados. Este dato permite realizar una comparación directa en cuanto a la diversidad (riqueza) de especies de individuos botánicos, aun cuando el número de individuos 
sea variable entre muestreos (el dato siempre es un valor entre 0 y 1 ; si todos los individuos de los muestreos fueran de especies diferentes, tendría un valor de 1 ; un valor de 0,5 significa una alta diversidad de especies) (Aguirre \& Endara, 2016). Es la cantidad total de especies presentes en una comunidad (Cano \& Stevenson, 2009).

Las Curvas especies-área se emplean para hallar el tamaño mínimo de la muestra, se asume que una vez que se alcanza una asíntota en el número de especies, el tamaño de muestra correspondiente es el área mínima de muestreo, que contiene una muestra representativa de la comunidad (Rosenzweig, 1995). Este a su vez permite inferir acerca del patrón de distribución agregado de las especies, en el control de los ensamblajes de especies en las comunidades vegetales (López \& Duque, 2010).

La Disimilitud Bray-Curtis (Bray-Curtis, 1957) se usa para cuantificar la disimilitud en la composición entre dos sitios diferentes basadas en conteos en cada sitio (Caranqui et al., 2016). Es una medida de la diferencia entre abundancia de cada especie presente (Argumedo \& Siqueiros, 2008).

$\mathrm{BC}_{\mathrm{ij}}=\left(2 \mathrm{C}_{\mathrm{ij}}\right) /\left(\mathrm{S}_{\mathrm{i}}+\mathrm{S}_{\mathrm{j}}\right)$

Donde: $\mathrm{C}_{\mathrm{ij}}=$ es la suma del valor menor para únicamente aquellas especies en común entre ambos sitios; $\mathrm{S}_{\mathrm{i}}$ y $\mathrm{S}_{\mathrm{j}}$ son el número total de especímenes contados en ambos sitios. $\mathrm{El}$ índice se reduce a $2 \mathrm{C} / 2=\mathrm{C}$, donde la abundancia en cada sitio se expresan como un porcentaje. El valor 1 significa que dos sitios tienen la misma composición (es decir que comparten todas las especies), y 0 significa que los dos sitios no comparten ninguna especie. En los sitios donde AC es intermedia (por ejemplo, $\mathrm{AC}=0,5$ ) este índice se diferencia de otros índices comúnmente utilizados (Caranqui, 2015; Caranqui etal., 2016)

Para la identificación de los nombres científicos se tomó como referencia bibliográfica de levantamientos florísticos realizados en el Bosque Universitario El Caimital (BUEC) (González, 2011; Lozada, 2008; Moret et al., 2010; Rangel, 2009) y la determinación de las muestras botánicas se realizó en el Laboratorio de Dendrología de la Facultad de Ciencias Forestales y Ambientales (FCFA), Universidad de Los Andes (ULA, Mérida, Venezuela), verificados y actualizados mediante la consulta en las bases de datos The Plant List (2013).

\section{Resultados}

Bosque no intervenido (BNI, 3 ha, Testigo) vs bosque intervenido (BI, 4 ha, fajas de enriquecimiento)
Análisis florístico

La tabla 1, muestra las 10 familias más importantes que resultaron en los levantamientos de las unidades de muestreo bosque no intervenido (BNI) versus bosque intervenido (BI). Se encontró una diferencia bajo la modalidad de censo de 54 individuos, 4 especies y 1 familia, siendo para el área basal valores con una diferencia del 0,5\%. De las familias con mayor Índice de Valor Familiar (IVF) presentes en bosque no intervenido (BNI) solo: Rubiacea (1 especies y 51 individuos); Anacardiaceae (3 especies y 34 individuos) y Polygonaceae (2 especies y 35 individuos), no coinciden dentro de las primeras 10 familias con el $\mathrm{IVF} \%$ en bosque intervenido (BI), apareciendo para este Bignoniaceae (3 especies y 64 individuos), Sapotaceae (2 especies y 28 individuos) y Combretaceae (1 especies y 31 individuos). Para ambos casos (bosque no intervenidoBNI y bosque intervenido-BI) las 10 primeras familias representan un $\mathrm{IVF} \%>69$.

El análisis del Índice de Valor de Importancia (IVI) e Índice de Importancia Ampliado (IIA) (tabla 2) muestra las 10 especies más importantes para ambos tratamientos, explicando los mayores valores de IIA\% $(55,97)$ e IVI\% $(46,12)$ para bosque intervenido (BI). Solo se observó una disminución de: 4 especies, 54 individuos y $0,31 \mathrm{~m}^{2}$ (área basal) del bosque intervenido (BI) (45 especies, 662 individuos, $\left.56,23 \mathrm{~m}^{2}\right)$ con respecto al bosque no intervenido (BNI) (49 especies, 716 individuos, 56,54 m²).

Dentro de las 10 primeras especies solo Trophis racemosa (con 66 individuos, un área basal de $5,26 \mathrm{~m}^{2} \mathrm{y}$ 4 individuos en el sotobosque), Triplaris americana (con 32 individuos, un área basal de 2,63 $\mathrm{m}^{2}$ y 2 individuos en el sotobosque) y Calycophillum candidissimum (con 51 individuos, un área basal de 4,3 $\mathrm{m}^{2} \mathrm{y}$ sin individuos en el sotobosque), no aparecen en el bosque intervenido (BI) siendo agregadas para este Tabebuia rosea (con 31 individuos, un área basal de 3,74 $\mathrm{m}^{2}$ y 2 individuos en el sotobosque, establecida en las fajas), Protium sp. (con 25 individuos, un área basal de $0,5 \mathrm{~m}^{2}$ y 5 individuos en el sotobosque) y Terminalia amazonia (con 31 individuos, un área basal de 2,96 $\mathrm{m}^{2}$ y 1 individuo en el sotobosque).

Cabe destacar la palma Attalea butyracea por los altos valores de IIA\% en ambos casos con 56 ind. (bosque no intervenido-BNI) y 96 ind. (bosque intervenido-BI) lo que reafirma las evidencias que existen de que esta especie es dominante en todo el pie de monte oriental de los Andes venezolanos, tanto en comunidades primarias como en las intervenidas (Franco \& Fölster, 1982; 
Tabla 1

Índice de Valor Familiar de la Composición Florística del bosque no intervenido (BNI, 3 ha, Testigo) versus bosque intervenido ( $\mathrm{Bl}, 4$ ha, fajas de enriquecimiento), El Caimital, Obispos, Barinas, Venezuela.

\begin{tabular}{|c|c|c|c|c|c|}
\hline Familias & Muestreo & $\mathrm{N}^{\circ}$ Especies & $\mathrm{N}^{\circ}$ Individuos & Área Basal (m2) & IVF\% \\
\hline Leguminosae & & 7 & 66 & 8,36 & 12,76 \\
\hline Moraceae & & 4 & 95 & 6,09 & 10,73 \\
\hline Arecaceae & & 2 & 64 & 6,66 & 8,27 \\
\hline Malvaceae & & 4 & 50 & 4,73 & 7,84 \\
\hline Euphorbiaceae & & 2 & 49 & 3,74 & 5,85 \\
\hline Rubiaceae & & 1 & 51 & 4,30 & 5,59 \\
\hline Anacardiaceae & Bosque No Intervenido (BNI) & 3 & 34 & 1,90 & 4,74 \\
\hline Meliaceae & & 2 & 45 & 2,03 & 4,65 \\
\hline Polygonaceae & & 2 & 35 & 2,74 & 4,61 \\
\hline Fabaceae & & 1 & 53 & 2,40 & 4,56 \\
\hline Sub-total 10 Familias & & 28 & 542 & 42,95 & 69,60 \\
\hline Sub-total 14 Familias & & 21 & 174 & 13,60 & 30,40 \\
\hline Total 24 Familias (BNI) & & 49 & 716 & 56,55 & 100,00 \\
\hline Arecaceae & & 3 & 119 & 12,94 & 15,89 \\
\hline Euphorbiaceae & & 2 & 78 & 11,46 & 12,21 \\
\hline Leguminosae & & 10 & 46 & 3,35 & 11,71 \\
\hline Bignoniaceae & & 3 & 64 & 4,79 & 8,29 \\
\hline Meliaceae & & 2 & 47 & 3,14 & 5,71 \\
\hline Malvaceae & & 3 & 26 & 2,41 & 4,96 \\
\hline Moraceae & Bosque Intervenido (BI) & 1 & 38 & 3,73 & 4,87 \\
\hline Sapotaceae & & 2 & 28 & 2,54 & 4,39 \\
\hline Fabaceae & & 1 & 51 & 1,70 & 4,32 \\
\hline Combretaceae & & 1 & 31 & 2,96 & 4,06 \\
\hline Sub-total 10 Familias & & 28 & 528 & 49,02 & 76,39 \\
\hline Sub-total 13 Familias & & 17 & 134 & 7,21 & 23,61 \\
\hline Total 23 Familias (BI) & & 45 & 662 & 56,23 & 100,00 \\
\hline
\end{tabular}

Nota: IVF\%: Índice de Valor Familiar porcentual.

Guevara, 2013; Kammesheidt et al., 2001; Lozada et al., 2009; Plonczak, 1993; Veillon, 1997).

En el mismo orden de ideas las especies Lonchocarpus pictus y Acalypha diversifolia, se mantienen en la tercera y octava posición, la primera con más de 50 ind. en el estrato superior para ambos casos y la segunda solo presente con individuos menores a $10 \mathrm{~cm}$ de (DAP), mientras que Inga sapindoides -disminuyendo de 17 individuos en bosque no intervenido (BNI) a tan solo 2 individuos en bosque intervenido (BI) - y Piper sp. (con tan solo representantes en el sotobosque para ambos casos) intercambian la cuarta $\mathrm{y}$ quinta posición respectivamente, siendo de igual forma para Myrcia guianensis -con representantes en el sotobosque para ambos casos, y tan solo 1 representante en el estrato superior en bosque intervenido (BI) que pasa de la décima a la novena posición-. 
Tabla 2

Índice de Valor de Importancia (IVI) e Importancia Ampliado (IIA) para las principales especies del bosque no intervenido (BNI, 3ha, Testigo) versus bosque intervenido (BI, 4 ha, fajas de enriquecimiento), El Caimital, Obispos, Barinas, Venezuela.

\begin{tabular}{|c|c|c|c|c|c|c|}
\hline Especie & Muestreo & $\mathbf{A i}$ & $\mathrm{Di}$ & $\mathrm{IVI} \%$ & As & $\| A \%$ \\
\hline Trophis racemosa & & 66 & 5,26 & 8,38 & 4 & 7,51 \\
\hline Attalea butyracea & & 56 & 6,38 & 8,33 & 4 & 7,16 \\
\hline Lonchocarpus pictus & & 53 & 2,40 & 6,08 & 2 & 4,89 \\
\hline Inga sapindoides & & 17 & 0,90 & 2,42 & 5 & 4,24 \\
\hline Piper sp. & & 0 & 0,00 & 0,00 & 8 & 3,99 \\
\hline Triplaris americana & & 32 & 2,63 & 4,37 & 2 & 3,87 \\
\hline Calycophillum candidissimum & Bosque No Intervenido & 51 & 4,30 & 6,32 & 0 & 3,79 \\
\hline Acalypha diversifolia & & 0 & 0,00 & 0,00 & 7 & 3,70 \\
\hline Hura crepitans & & 33 & 2,21 & 4,41 & 1 & 3,27 \\
\hline Myrcia guianensis & & 0 & 0,00 & 0,00 & 5 & 3,11 \\
\hline Sub-total 10 especies & & 308 & 24,07 & 40,32 & 38 & 45,53 \\
\hline Sub-total 39 especies & & 408 & 32,47 & 59,68 & 30 & 54,47 \\
\hline Total 49 especies & & 716 & 56,54 & 100,00 & 68 & 100,00 \\
\hline Attalea butyracea & & 96 & 11,98 & 14,87 & 7 & 12,07 \\
\hline Hura crepitans & & 69 & 10,26 & 11,99 & 2 & 8,09 \\
\hline Lonchocarpus pictus & & 51 & 1,70 & 5,75 & 9 & 7,50 \\
\hline Piper sp. & & 0 & 0,00 & 0,00 & 14 & 5,14 \\
\hline Inga sapindoides & & 2 & 0,04 & 0,29 & 9 & 4,22 \\
\hline Tabebuia rosea & & 31 & 3,74 & 5,20 & 2 & 4,02 \\
\hline Protium sp. & Bosque Intervenido & 25 & 0,50 & 2,64 & 5 & 3,83 \\
\hline Acalypha diversifolia & & 0 & 0,00 & 0,00 & 9 & 3,82 \\
\hline Myrcia guianensis & & 1 & 0,01 & 0,14 & 8 & 3,68 \\
\hline Terminalia amazonia & & 31 & 2,96 & 5,24 & 1 & 3,59 \\
\hline Subtotal 10 especies & & 306 & 31,18 & 46,12 & 66 & 55,97 \\
\hline Subtotal 35 especies & & 356 & 25,04 & 53,88 & 26 & 44,03 \\
\hline Total 45 especies & & 662 & 56,23 & 100,00 & 92 & 100,00 \\
\hline
\end{tabular}

Nota: Ai: Abundancia en el estrato superior; Di: Dominancia en el estrato superior; IVI\%: Índice de Valor de Importancia porcentual; As: Abundancia en el sotobosque; IIA\%: Índice de Importancia Ampliado porcentual.

Cabe resaltar que la especie Hura crepitans aparece en segundo lugar para el bosque intervenido (BI) debido a que esta fue establecida dentro de las fajas de enriquecimiento (con 69 representantes, un área basal de 10,26 $\mathrm{m}^{2}$ y tan solo dos representantes en el sotobosque), de igual modo se establece dentro de las 10 primeras especies para el bosque no intervenido (BNI) (con 33 representantes, un área basal de 2,21 $\mathrm{m}^{2}$ y tan solo 1 representante en el sotobosque).
Para los individuos con diámetros menores que $10 \mathrm{~cm}$ se registró para el bosque intervenido (BI) un total de 92 ind. $/ 180 \mathrm{~m}^{2}$ (para las $3 \mathrm{ha}$ ) (tabla 2), lo que representa un promedio de 30,6 ind. $/ 60 \mathrm{~m}^{2}$ (para $1 \mathrm{ha}$ ) equivalentes a 5.100 ind./ha, concentrados entre las primeras 10 especies, siendo las más numerosas Piper sp. (14 ind.), Lonchocarpus pictus, Inga sapindoides y Acalypha diversifolia con (9 ind.) respectivamente. Para el bosque no intervenido (BNI) son 

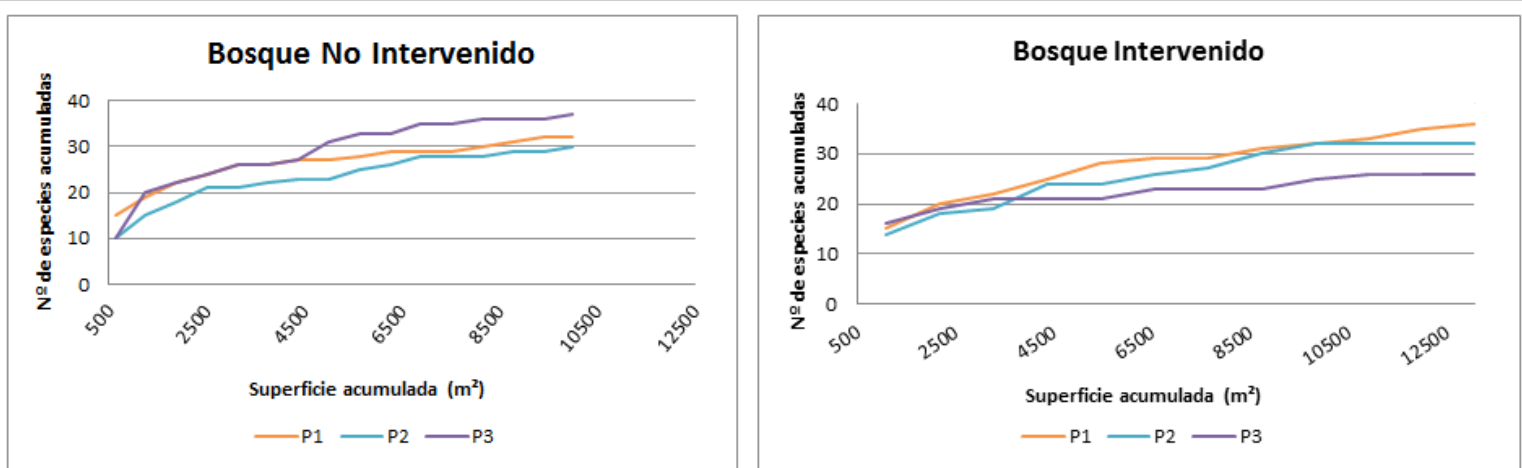

Figura 2. Curvas Especie-Área: a) bosque no intervenido (BNI, 3 ha, Testigo, izquierda). b) Bosque intervenido (BI, 4 ha, fajas de enriquecimiento, derecha) El Caimital, Obispos, Barinas, Venezuela. Nota: P1: unidad de muestreo 1; P2: unidad de muestreo 2; P3: unidad de muestreo $3 .$.

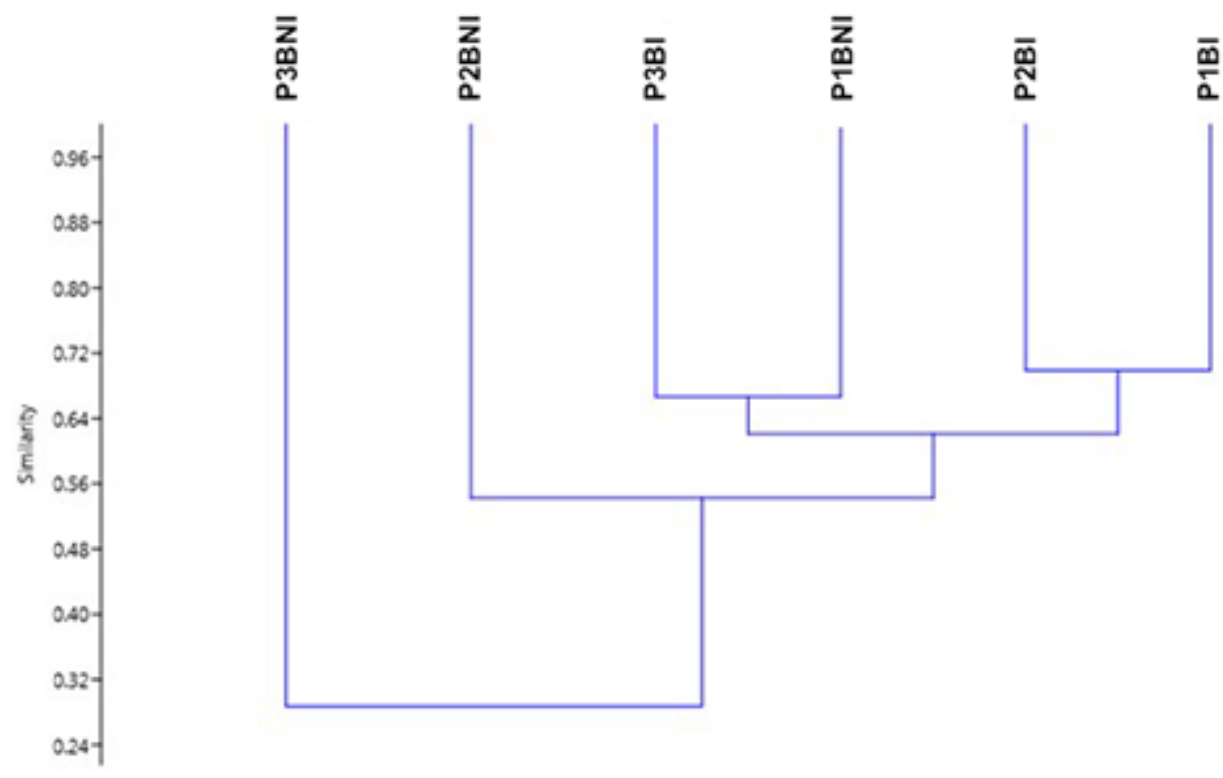

Figura 3. Dendrograma de similitud-disimilitud (Bray-Curtis) para las comunidades potenciales para las áreas de (3 ha, bosque no intervenido-Testigo BNI y 4ha, bosque intervenido, BI - fajas de enriquecimiento) El Caimital, Obispos, Barinas, Venezuela. Nota: P1BNI: unidad de muestreo 1 en bosque no intervenido; P2BNI: unidad de muestreo 2 en bosque no intervenido; P3BNI: unidad de muestreo 3 en bosque no intervenido; P1BI: unidad de muestreo 1 en bosque intervenido; P2BI: unidad de muestreo 2 en bosque intervenido; P3BI: unidad de muestreo 3 en bosque intervenido.

registrados un total de 68 ind. $/ 180 \mathrm{~m}^{2}$ (para las $3 \mathrm{ha}$ ) (tabla 2), lo que representa un promedio de 22,6 ind. $/ 60 \mathrm{~m}^{2}$ (para 1 ha) equivalentes a 3.767 ind./ha, concentrados entre las primeras 10 especies, mostrándose en primer lugar Piper sp. (8 ind.), seguida de Acalypha diversifolia (7 ind.), Inga sapindoides y Myrcia guianensis con (5 ind. c/u).

Las curvas de especies áreas de los individuos con DAP $\geq 10 \mathrm{~cm}$ (figura 2), considerando todas las unidades de muestreo en conjunto y en una misma posición fisiográfica (banco), muestra que para ambos casos (bosque no intervenido-BNI y bosque intervenido-BI), un área de $5.500 \mathrm{~m}^{2}$ es un área relativamente adecuada para el estudio de la vegetación a través de unidades de muestreo, ya que un aumento en el 10\% en el área levantada genera un incremento inferior al 10\% en el número de especies (criterio de Cain, 1938 citado por Muller-Dombois \& Ellenberg, 1974).

El dendrograma de similitud Bray-Curtis (figura 3) indica que a excepción de la unidad de muestreo (P3ESTES-o Estrato superior-testigo) existe una similaridad en el resto de las unidades de muestreo superior al $50 \%$, y solo mayor al $30 \%$ entre todas las unidades de muestreo (bosque no intervenido-BNI y bosque intervenido-BI), estas diferencias pueden deberse a distinciones en el 

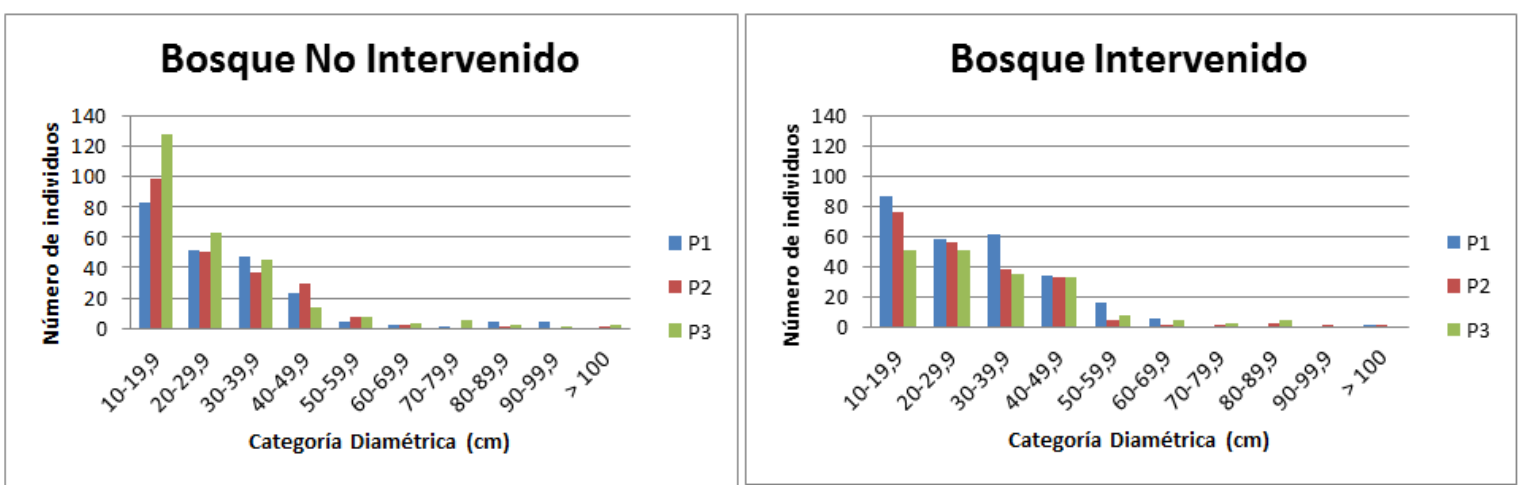

Figura 4. Distribución por Clases Diamétricas: a) Bosqueno intervenido (BNI, 3 ha, Testigo, izquierda). b) Bosque intervenido (BI, 4 ha, fajas de enriquecimiento, derecha) El Caimital, Obispos, Barinas, Venezuela.

área en cuanto a características del suelo que afectan la distribución de las especies presentes.

El BUEC forma parte de las sabanas de banco, bajío y estero de las planicies aluviales recientes, con una geomorfología aluvial típica de algunas zonas de los llanos occidentales venezolanos influenciada por la dinámica del río Yuca que se encuentra al norte de este bosque (Rangel et al., 2021). Los suelos del bosque Caimital, como corresponde en general a los llanos occidentales, son de origen aluvial y aluvio lacustrino, formado por materiales arrastrados por las aguas desde las formaciones montañosas vecinas. Pueden denominarse como Regosoles aluviales en procesos de ferretización y que tienden, como clímax, a la laterita (Moret et al., 2008).

La distribución por categorías diamétricas (figura 4) se presenta en forma de "J" invertida para ambos casos (bosque no intervenido-BNI y bosque intervenido-BI), consiguiendo que la mayor abundancia de individuos oscilan en la primera categoría diamétrica $(10-19,9 \mathrm{~cm})$ con una diferencia del 10,83\% (bosque no intervenido$\mathrm{BNI})$ superior al bosque intervenido (BI), seguido de la segunda categoría diamétrica (20-29.9 cm) con un 2,02 $\%$ más para bosque intervenido $(\mathrm{BI})$ alcanzando así, para ambos casos más del $50 \%$ de individuos solo en estas dos categorías.

\section{Análisis de diversidad arbórea}

Los resultados para la biodiversidad alfa $(\alpha)$ por parcela (bosque no intervenido-BNI versus bosque intervenido-BI) y en promedio dentro de la misma condición fisiográfica (banco), incluyendo todas las categorías diamétricas se presentan en la tabla 3. En los promedios para los estratos superiores todos los índices muestran valores similares para ambos casos (bosque no intervenido-BNI y bosque intervenido-BI). Las mayores riquezas se encuentran en la unidad de muestreo (P3) (bosque no intervenido-BNI=37) y unidad de muestreo (P1) (bosque intervenido-BI=36). A nivel de abundancia a excepción de la unidad de muestreo (P1), los mayores valores son encontrados en el bosque no intervenido (BNI). Es en la unidad de muestreo (P3)-bosque no intervenido (BNI) versus unidad de muestreo (P3)-bosque intervenido (BI) donde se halló diferencias visibles de los índices Fisher Alpha y Margalef con $+3,38$ y $+1,64$ respectivamente. Siendo el coeficiente de mezcla para ambos casos (bosque no intervenido-BNI y bosque intervenido-BI) igual para todas las unidades de muestreo, ya que se incorpora una especie por cada siete muestreadas. Para los individuos con un DAP $<10 \mathrm{~cm}$ (tabla 3 ), para los promedios en el bosque intervenido (BI) existe una mayor diferencia en abundancia de +8 . Siendo para los índices Fisher Alpha y Margalef donde se encontró las mayores diferencias en sus valores (bosque no intervenido-BNI versus bosque intervenido-BI).

\section{Análisis de similitud florístico}

Los resultados encontrados para el Índice de similitud Sorensen, en las tres (3) unidades de muestreo de bosque no intervenido (BNI) y bosque intervenido (BI) (tabla 4), muestran que, en bosque intervenido (BNI) existe menor similitud entre unidades de muestreo, no logrando superar el $45 \%$, a pesar de encontrarse bajo la misma condición fisiográfica. El bosque intervenido (BI) es el más homogéneo por superar hasta una similitud superior al $70 \%$ entre las unidades de muestreo P2 y P3, lo que indica que el área seleccionada fue la más adecuada para materializar la evaluación. 
Tabla 3

Índice de Diversidad alfa por parcela, para las diferentes especificaciones diamétricas del bosque no intervenido (BNI, 3ha, Testigo) versus bosque intervenido (BI, 4 ha, fajas de enriquecimiento), El Caimital, Obispos, Barinas, Venezuela.

\begin{tabular}{|c|c|c|c|c|c|c|}
\hline Índice & Estrato & Muestreo & P1 & P2 & P3 & Promedio \\
\hline Riqueza & \multirow{12}{*}{$\begin{array}{l}\text { Estrato Superior } \\
(\mathrm{DAP} \geq 10 \mathrm{~cm})\end{array}$} & \multirow{6}{*}{$\mathrm{BNI}$} & 32,00 & 30,00 & 37,00 & 33,00 \\
\hline Abundancia & & & 219,00 & 226,00 & 271,00 & 238,66 \\
\hline Fisher_alpha & & & 10,32 & 9,28 & 11,58 & 10,39 \\
\hline Margalef & & & 5,75 & 5,35 & 6,43 & 5,84 \\
\hline Simpson_1-D & & & 0,93 & 0,92 & 0,93 & 0,92 \\
\hline Shannon_H & & & 2,96 & 2,84 & 2,98 & 2,92 \\
\hline Riqueza & & \multirow{6}{*}{$\mathrm{Bl}$} & 36,00 & 32,00 & 26,00 & 31,33 \\
\hline Abundancia & & & 262,00 & 208,00 & 187,00 & 219,00 \\
\hline Fisher_alpha & & & 11,30 & 10,56 & 8,20 & 10,02 \\
\hline Margalef & & & 6,27 & 5,81 & 4,78 & 5,62 \\
\hline Simpson_1-D & & & 0,95 & 0,93 & 0,91 & 0,92 \\
\hline Shannon_H & & & 3,21 & 2,99 & 2,71 & 2,96 \\
\hline Riqueza & \multirow{12}{*}{$\begin{array}{l}\text { Sotobosque } \\
(\mathrm{DAP}<10 \mathrm{~cm})\end{array}$} & \multirow{6}{*}{$\mathrm{BNI}$} & 20,00 & 16,00 & 11,00 & 15,66 \\
\hline Abundancia & & & 38,00 & 16,00 & 14,00 & 22,66 \\
\hline Fisher_alpha & & & 17,08 & 0,00 & 23,69 & 13,59 \\
\hline Margalef & & & 5,22 & 5,41 & 3,79 & 4,80 \\
\hline Simpson_1-D & & & 0,93 & 0,94 & 0,90 & 0,92 \\
\hline Shannon_H & & & 2,82 & 2,77 & 2,34 & 2,64 \\
\hline Riqueza & & \multirow{6}{*}{$\mathrm{Bl}$} & 15,00 & 16,00 & 16,00 & 15,66 \\
\hline Abundancia & & & 29,00 & 32,00 & 31,00 & 30,66 \\
\hline Fisher_alpha & & & 12,50 & 12,70 & 13,29 & 12,84 \\
\hline Margalef & & & 4,16 & 4,33 & 4,37 & 4,28 \\
\hline Simpson_1-D & & & 0,92 & 0,92 & 0,91 & 0,91 \\
\hline Shannon_H & & & 2,58 & 2,66 & 2,59 & 2,61 \\
\hline
\end{tabular}

Nota: BNI: bosque no intervenido; DAP: Diámetro a la altura de pecho en m; BI: Bosque intervenido; P1: unidad de muestreo 1; P2: unidad de muestreo2; P3: unidad de muestreo 3.

\section{Avance de la sucesión secundaria}

Analizando la composición florística del bosque original en comparación con el bosque intervenido, son evidentes los cambios que se presentan con relación a la abundancia (tabla 2), ya que en el bosque primario las especies más representativas son: Trophis racemosa con 66 ind., Attalea butyracea con 56 ind., Lonchocarpus pictus con 53 ind. y Calycophillum candidissimum con 51 ind. dentro de las 10 especies más importantes, siendo en el bosque secundario un incremento notorio de la palma Attalea butyracea con 96 ind., apareciendo en segundo lugar Hura crepitans con 69 ind. y Lonchocarpus pictus con 51 ind. mantiene un valor similar.

Las especies Trophis racemosa, Triplaris americana, Calycophillum candidissimum desaparecen dentro de las 10 primeras especies importantes para el bosque intervenido. Una causa que probablemente está influyendo en el aumento de la abundancia de Attalea butyracea es la existencia de una mayor formación de claros dentro del bosque intervenido. Mientras que para la especie 


\section{Tabla 4}

Índice de similitud Sorensen del bosque no intervenido (BNI, 3 ha, Testigo) versus bosque intervenido ( $\mathrm{Bl}$, 4ha, fajas de enriquecimiento), El Caimital, Obispos, Barinas, Venezuela.

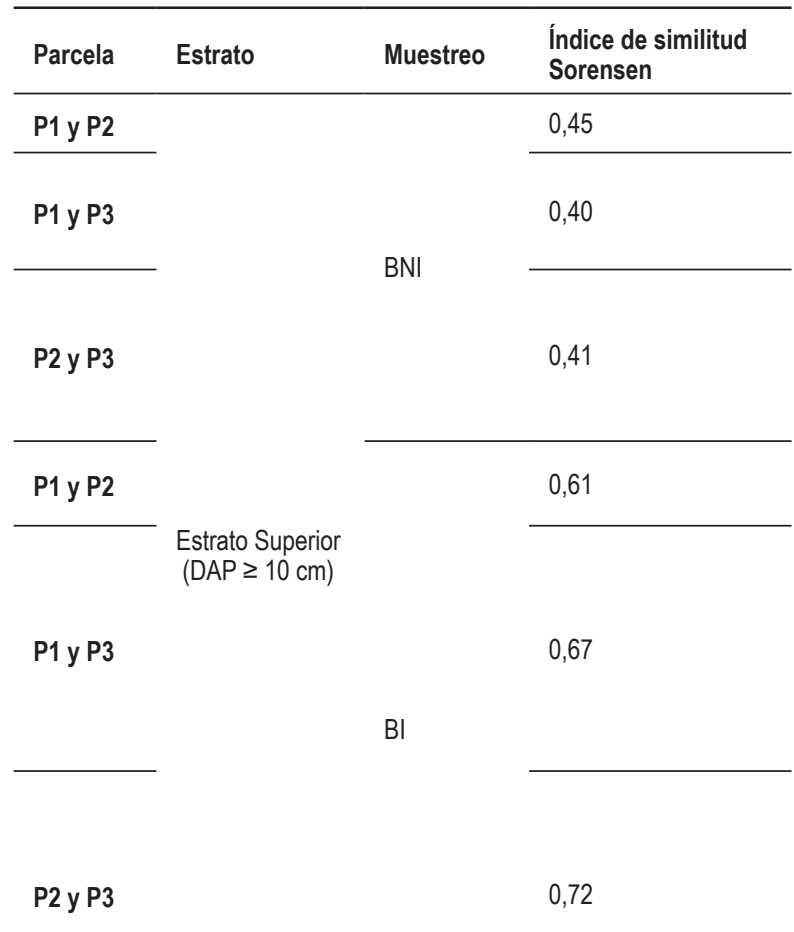

Nota: BNI: Bosque no intervenido; DAP: Diámetro a la altura de pecho en m; BI: Bosque intervenido; P1: unidad de muestreo1; P2: unidad de muestreo2; P3: unidad de muestreo3.

Hura crepitans su aparición se debe en gran parte a que esta fue plantada dentro de las fajas de enriquecimiento. Siendo las especies A. butyraceae, Lonchocarpus pictus, Inga sapindoides, Piper sp., Acalypha diversifolia, Hura crepitans y Myrcia guianensis constantes dentro de las 10 primeras especies en ambos tipos de bosques.

Con relación a la altura, cabe destacar que en el bosque intervenido (BI) se registró una altura promedio de 14,1 m observándose algunos árboles emergentes con alturas superiores a los 20 metros para las especies Luehea cymulosa, Hura crepitans, Triplaris americana, Tabebuia rosea, Astronium graveolens, Jacaranda obtusifolia, Lonchocarpus margaritensis, Sapium glandulosum, Trophis racemosa, Albizia saman, Melicoccus bijugatus, y en el bosque no intervenido (BNI) el promedio en altura fue de 15,9 m, observándose algunos árboles emergentes con alturas superiores a los 20 metros para las especies Trophis racemosa, Cordia bicolor, Astronium graveolens, Triplaris americana, Luehea cymulosa, Lonchocarpus pictus, Albizia niopoides, Terminalia amazonia, Hura crepitans, Prionostemma aspera, Pterocarpus acapulcensis.

En la distribución diamétrica de las especies evaluadas, el $75 \%$ de los individuos se concentraban en las categorías de 10-49,9 cm en ambos bosques (bosque no intervenido-BNI y bosque intervenido-BI), la especie Trophis racemosa es la mejor distribuida para el bosque no intervenido (BNI) presente con 56 individuos y para el bosque intervenido (BI) la especie Lonchocarpus pictus la mejor distribuida con 51 individuos.

Sin embargo, al analizar las categorías superiores DAP $>50$ $\mathrm{cm}$, se observa que la categoría diamétrica $90-99,9 \mathrm{~cm}$, presenta una mayor cantidad de individuos, la especie Pterocarpus acapulcensis es la que se encuentra mejor distribuida en el bosque no intervenido (BNI), y para el bosque intervenido (BI) la categoría diamétrica más numerosa es 50-59,9 cm siendo la especie la mejor distribuida Hura crepitans.

Analizando el número total de individuos en los bosques estudiados, se observó que para el bosque no intervenido (BNI) actualmente existen (716 ind. - en 49 especies) mientras que para el bosque intervenido (BI) (662 ind. en 45 especies), lo que indica que ciertamente la sucesión secundaria ha logrado avanzar, después de 57 años).

\section{Conclusiones}

Evidentemente el Bosque Universitario "El Caimital" se ha visto influenciado por el sistema silvicultural "fajas de enriquecimiento" por haberse encontrado a través del censo realizado mayores especies comerciales en el lote donde se encuentra dicho sistema, proporcionándole al mismo un gran valor económico, a pesar de que se encuentren menos individuos que en el bosque no intervenido.

En los últimos 57 años no se han observado cambios muy marcados en la composición florística, ya que se encontró un total de 45 especies arbóreas, agrupadas en 23 familias para el bosque intervenido (BI), las Arecaceae, Euphorbiaceae, Leguminoceae, Bignonaceae, Meliaceae, Malvaceae, Moraceae, Sapotaceae, Fabaceae y Combretaceae son las más importantes respectivamente, con una semejanza del 40 $\%$ respecto al bosque primario. Los valores para el bosque no intervenido (BNI) son: 49 especies, agrupadas en 24 familias destacando entre ellas Leguminoceae, Moraceae, Arecaceae, Malvaceae, Euphorbiaceae, Rubiaceae (no aparece dentro de las 10 primeras en bosque intervenido$\mathrm{BI}$ ), Anacardiaceae (no aparece dentro de las 10 primeras en bosque intervenido-BI), Meliaceae, Polygonaceae (no 
aparece dentro de las 10 primeras en bosque intervenidoBI) y Fabaceae respectivamente.

Según el IVI e IIA, las especies más importantes del bosque primario son: Trophis racemosa, Attalea butyraceae, Lonchocarpus pictus, Inga sapindoides, Piper sp., Triplaris americana, Calycophillum candidissimum, Acalypha diversifolia, Hura crepitans y Myrcia guianensis. Para el bosque secundario avanzado las especies Attalea butyracea, Hura crepitans, Lonchocarpus pictus, Piper sp., Inga sapindoides, Acalypha diversifolia y Myrcia guianensis se muestran dentro de las más importantes, siendo Tabebuia rosea, Protium sp., y Terminalia amazonia encontradas solo para bosque intervenido (BI).

A través de los índices ecológicos se logró comprobar que la plantación por fajas de enriquecimiento logra mantener la diversidad del bosque ya que los valores arrojados para los diferentes índices nos revelan que, la zona de establecimiento es una zona de alta diversidad por haber obtenido valores mayores a 5,0 en cuanto al índice de Margalef, lo cual es indicativo de alta biodiversidad.

Por otro lado, el levantamiento realizado en bosque intervenido (BI) nos señala la dominancia de una especie sobre las demás mostrándonos en cuanto al índice de Simpson valores cercanos a 1 , con esto se explica la dominancia que tiene la especie Hura crepitans sobre las demás, mientras que para el bosque no intervenido (BNI) está dominancia se presenta con la especie Attalea butyraceae.

Para las categorías diamétricas, podemos observar que ambos lotes evaluados se presentan en forma de "J" invertida lo cual es característico de los bosques naturales. En la manera en que se encuentran la mayor cantidad de individuos en categorías menores a $20 \mathrm{~cm}$ de (DAP), nos que esta cantidad de individuos son producto de regeneración natural y del imput de semillas que las especies comerciales establecidas han arrojado, siendo la especie Hura crepitans la más eficiente y adaptable en este bosque, ya que es la que tienen una mayor abundancia y se encuentran en categorías de hasta $100 \mathrm{~cm}$ de (DAP).

Por falta de información (monitoreo), no se puede caracterizar de manera adecuada las tendencias de la regeneración natural (mortalidad y reclutamiento), la posible importancia de una especie (densidad y área basal).

En cuanto a su distribución por categorías diamétricas, resalta la especie Hura crepitans por encontrarse en todas las clases y el resto de las especies en categorías menores a $70 \mathrm{~cm}$. Esto sigue demostrando que Hura crepitans es la especie que mejor se adapta en este tipo de bosque.

Es lamentable que, por falta de seguimiento y mantenimiento al sistema, no se tenga con certeza a cuál de las fajas de enriquecimiento pertenecen cada una de las especies comerciales encontradas.

La variabilidad que se encontró en los individuos evaluados puede atribuirse a factores como: a) a pesar de que se tienen referencias de aclareos (Vincent, 1969) y replanteos (Benitez et al., 1987) para evitar la competencia temprana entre los individuos plantados no existe el seguimiento de estos para afirmar si estas técnicas fueron adecuadas o no. b) En observaciones realizadas en campo se considera que existen diferencias en el relieve y suelos. c) El ataque observado de Hypsipylla grandella (1973) contra la especie Swietenia macrophylla casi en la totalidad de su población corrobora el mantenimiento inadecuado a las plantaciones. d) Puede que el distanciamiento implementado entre plantas desfavorezca a algunas de las especies. e) Ausencia de un sistema de tipificación de áreas que permita ubicar a cada especie de acuerdo a sus requerimientos ecológicos.

Se hace imposible identificar con certeza a que fajas pertenecen los 131 árboles encontrados de las especies establecidas, solo la faja 1 y 15 pueden detallarse en una transepta aproximada de 250 metros lineales mientras que el resto de las fajas solo a unos casos metros se puede observar con detenimiento.

El BUEC se posiciona como uno de los parches más importantes dentro del bosque seco tropical a pesar de poca extensión, debido a sus características de riqueza florística, almacenamiento de biomasa, estructura, dinámica y composición, mostrando un proceso sucesional que está permitiendo la recuperación del ecosistema.

\section{Referencias}

Aguirre, X. \& Endara, A. (2016). Diversidad de flora vascular del Chocó Andino en el área de Selva Virgen, Ecuador. Enfoque UTE, 7(2), 82-96. https:// doi.org/10.29019/enfoqueute.v7n2.97

Álvarez, C. \& Lara, A. (2008). Crecimiento de una plantación joven en fajas con especies nativas en la Cordillera de Los Andes de la provincia de Valdivia. BOSQUE 29(3), 181-191. https://doi.org/10.4067/ s0717-92002008000300001 
Alvis, J. (2009). Análisis estructural de un bosque natural localizado en zona rural del municipio de Popayán. Biotecnología en el sector Agropecuario y Agroindustrial, 7(1), 115-122. http://www.scielo. org.co/scielo.php?script=sci_arttext\&pid=S169235612009000100013\&Ing=en\&tlng=es

Argumedo, U. \& Siqueiros, D. (2008). Cambios en la estructura de la asociación de diatomeas epifitas de Macrocystis pyrifera (L.) C. Ag. Acta botánica mexicana, 82, 43-66. http://www.scielo. org. $\mathrm{mx} / \mathrm{scielo}$.php? script=sci_arttext\&pid=S018771512008000100005\&Ing=es\&tlng=es

Benítez, D., Espinoza, E., \& Prado, L. (1987). Evaluación y aplicación con fines de manejo de un ensayo de enriquecimiento en fajas en un bosque deciduo tropical Caimital Barrancas Edo. Barinas. Universidad de Los Andes. Mérida, Venezuela.

Bernal, J. (1967). Estudio ecológico del bosque Caimital. Revista Forestal Venezolana, 5, 47-82.

Bray, J. R. \& Curtis. J. T. (1957). An ordination of the upland forest communities of southern. Wisconsin. Ecological monographs, 27(4), 325-349. https://doi. org/10.2307/1942268

Bustamante, M., (2009). Biodiversidad de plantas en el borde amazónico putumayense: i. inventario. CienciAgro, 1(4), 129-137. http://www. revistasbolivianas.org.bo/scielo.php?script=sci_ arttext\&pid=S2072-14042009000100005\&\&Ing=es $\& n r m=$ iso.

Cabrera, M., Segura, E., Segura, J., \& Tierres, J. (2018). Composición y diversidad de la vegetación arbórea, un instrumento de gestión turística, caso: parque ecológico recreacional lago Lagrio (Perla) Sucumbíos - Ecuador. Revista Cubana de Ciencias Forestales, 6(3), 327-340. http://www.scielo.sld. $\mathrm{cu} /$ scielo.php?script=sci_arttext\&pid=S2310$34692018000300327 \&$ Ing $=$ es\&tlng=es

Campo, A. \& Duval V. (2014). Diversidad y valor de importancia para la conservación de la vegetación natural. Parque Nacional Lihué Calel (Argentina). Anales de Geografía de la Universidad Complutense, 34(2), 25-42. https://doi.org/10.5209/rev_ AGUC.2014.v34.n2.47071
Cano, A. \& Stevenson, P. (2009). Diversidad y composición florística de tres tipos de bosque de la estación biológica Caparú, Vaupés. Revista Colombia Forestal, 12(1), 63-80. http://www.scielo. org.co/scielo.php?script=sci arttext\&pid=S012007392009000100006\&Ing=en\&tlng=es

Caranqui, J. (2015). Composición y diversidad de especies arbóreas en transectos de localidades del bosque siempreverde de tierras bajas del Ecuador. Enfoque UTE, 6(3), 96-105. https://doi.org/10.29019/ enfoqueute.v6n3.72

Caranqui, J., Lozano, P., \& Reyes, J. (2016). Composición y diversidad florística de los páramos en la Reserva de Producción de Fauna Chimborazo, Ecuador. Enfoque UTE, 7(1), 33-45. https://doi.org/10.29019/ enfoqueute.v $7 \mathrm{n} 1.86$

Corredor, J. (2001). Silvicultura Tropical. Consejo de publicaciones. Consejo de Desarrollo -científico, Humanístico y Tecnológico. Universidad de Los Andes.

Curtis, J. T., \& McIntosh, R. P. (1951). An upland forest continuum in the prairie-forest border region of Wisconsin. Ecology, 32(3), 476-496. https://doi. org/10.2307/1931725

Fisher, R.A., Cobet, AS., \& Williams, C.B. (1943). The relation between the number of species and the number of individuals in a random sample of an animal population. Journal of Animal Ecology, 12(1), 42-58. https://doi.org/10.2307/1411

Flores, Y., Ugalde, L., Galloway, G., \& Carrera, F. (2004). Crecimiento y productividad de plantaciones de seis especies forestales en nativas de 20 años de edad en el bosque Alexander Von Humbolt, Amazonia Peruana. CATIE. Soluciones para el ambiente y desarrollo. Repositorio Institucional. Recursos Naturales y Ambiente, 41-42-43. http://hdl.handle. net/11554/9786

Franco, W., \& Folster, H. (1982). Estudio de los suelos de la Reserva Forestal Caparo, Estado Barinas, con énfasis en las propiedades fisicas y régimen hídrico de los mismos. VII Congreso Venezolano de Ciencias del Suelo. San Cristóbal, Venezuela. 
González, J. (2011). Distribución espacial de Paquira quinata (saqui-saqui) y muestreo de la vegetación en el Sector «B» del bosque universitario «el caimital» estado Barinas, Venezuela. Universidad de Los Andes.

Guevara, J. (2013). Recursos Fitogenéticos y Relaciones Florísticas de la Flórula arbórea de las Comunidades Forestales en la Estación Experimental Caparo, Estado Barinas.

Iannacone, J., Mansilla, J., \& Ventura, K. (2003). Macroinvertebrados en las lagunas de puerto viejo, Lima - Perú. Ecología Aplicada, 2(1), 116-124. https://doi.org/10.21704/rea.v2i1-2.258

Kammesheidt, L., Lezama, A., Franco, W., \& Plonczak, M. (2001). History of logging and silvicultural treatments in the western Venezuela plain forests and prospect for sustainable forest management. Forest Ecology and Management, 148(1-3), 1-20. https:// doi.org/10.1016/s0378-1127(00)00529-6

Konrad, V. (1961). Plan de ordenación del bosque experimental "Caimital" propiedad de la Universidad de Los Andes. Universidad de Los Andes.

Lamprecht, H.L. (1990). Silvicultura en los trópicos: los ecosistemas forestales en los bosques tropicales y sus especies arbóreas; posibilidades y métodos para un aprovechamiento sostenido. TZ-Verlag-Ges.

Lirio, R., Lozada, J., Mora, A., \& Lugo, L. (2010). Efecto de borde en sistemas de enriquecimiento en fajas, Reserva Forestal de Imataca, Venezuela. Revista Forestal Venezolana. 55(1), 61-73. http:// www.researchgate.net/publication/236596854 Efecto_de_borde_en_sistemas_de_enriquecimiento en_fajas_Reserva_Forestal_Imataca_Venezuela

López, W., \& Duque, A. (2010). Patrones de diversidad alfa en tres fragmentos de bosques montanos en la región norte de los Andes, Colombia. Revista de Biología Tropical, 58(1), 483-498. https://doi. org/10.15517/rbt.v58i1.5223

Lozada, J.R. (2008). Sucesión vegetal en bosques aprovechados de la Reserva Forestal Caparoy Reserva Forestal Imataca, Venezuela. [Tesis doctoral. Valencia, España]. https://repositorioslatinoamericanos.uchile. $\mathrm{cl} /$ handle/2250/214257? show=full
Lozada, J. (2010). Consideraciones metodológicas sobre los estudios de comunidades forestales. Revista Forestal Venezolana, 54(1), 77-88. https:// www.researchgate.net/publication/47512174 Consideraciones_metodologicas_sobre_los_estudios de_comunidades_forestales

Lozada, J., Arends, E., Sánchez, D., Villareal, A., Soriano, P., \& Costa, M. (2009). Cambios de Composición Florística en Bosques Aprovechados de la Estación Experimental Caparo. Revista Forestal Latinoamericana, 24(2), 35-50. https://www. researchgate.net/publication/236651452_Cambios_de_ composicion_floristica_en_bosques_aprovechados de_la_Estacion_Experimental_Caparo

Lozada, J., Guevara, J., Hernández, C., Soriano, P., \& Costa, M. (2011). Los bosques de la zona central de la Reserva Forestal de IMATACA, estado BolívarVenezuela. BioLlania, 10, 47-62. http://www.researchgate. netpublication/236596832_Los_bosques_de_la_zona_central de la Reserva_Forestal_Imataca_Estado_Bolivar_Venezuela

Lozada, J., Moreno, J., \& Suescun, R. (2003). Plantaciones en Fajas de Enriquecimiento. Experiencias en 4 unidades de manejo forestal de la Guayana venezolana. Interciencia, 28(10), 568-575. http:/ve.scielo.org/scielo.php?script=sci_arttext\&pid=S0378$18442003001000004 \& \operatorname{lng}=$ es\&tlng=en

Magurran, A.E. (1988). Ecological Diversity and its Measurement. Princenton University Press. https:// doi.org/10.1007/978-94-015-7358-0

Margalef, R. (1977). Ecología. Ediciones Omega.

McIntosh, R. I. (1967). An index of diversity and the relation of certain concepts to diversity. Ecology, 48(3), 392-404. https://doi.org/10.2307/1932674

Montilla, M., \& Rivas, M. (1987). Estudio sobre la Regeneración Natural del Bosque Secundario Tropófito Macrotérmico (Caimital Edo. Barinas). Universidad de Los Andes.

Mora, C., Pérez, J., Rodríguez, E., Rubio, E., YerenaYamallel, J., \& González, M. (2013). Efecto de la ganadería en la composición y diversidad arbórea y arbustiva del matorral espinoso Tamaulipeco. Revista Mexicana de Ciencias Forestales, 4(17), 124-137. http://www.scielo.org.mx/scielo.php?script=sci_ arttext\&pid=S2007-11322013000300012 
Moret, A., Plonczak, M., Jerez, M., Garay, V., Valera, L., Ramírez, N., Hernández, D., \& Mora, A. (2010). Variaciones en la composición florística de tipos de bosque asociados con Pachira quinata (Jacq.) W.S. Alverson en el Bosque Universitario "El Caimital", Barinas, Venezuela. Revista Forestal Venezolana, 54(1), 54-63. https:// www.researchgate.net/publication/291356251 Moret AY M Plonczak M Jerez V Garay L Valera_N_Ramirez_D_Hernandez_A_Mora_2010 Variacion_en_la_composicion_floristica_da_tipos de_bosques_asociados_con_Pachira_quinata Jacq_W_S_Alverson_en_el_bosque_U

Moret, A., Valera, L., Mora, A., Garay, V., Jerez, M., Plonczak, M., Ramírez, N., \& Hernández, D. (2008). Estructura horizontal y vertical de Pachira quinata (Jacq.) W.S Alverson, (Bombacaceae) en el bosque universitario El Caimital, Barinas, Venezuela. Ecotrópicos, 21(2), 62-75. https://www. researchgate.net/publication/234052496_Estructura horizontal_y_vertical_de_Pachira_quinata_Jacq WS_AlversonBombacaceae_en_el_bosque_ universitario_El_Caimital_Barinas_Venezuela

Mori, S., B. Boom, A. de Carvalho, \& T. dos Santos. (1983). Southern Bahian moist forest. The Botanical Review, 49, 155-232. https://link.springer.com/ article/10.1007/BF02861011

Muller-Dombois, D., \& Ellenberg, H. (1974). Aims and methods of vegetation Ecology. John Wiley \& Sons.

Plonczak, M. (1993). Estructura y Dinámica de Desarrollo de Bosques Naturales Manejados Bajo la Modalidad de Concesiones en los Llanos Occidentales de Venezuela. Instituto Forestal Latinoamericano.

Rangel, R., Salcedo, P. , \& Gómez, A. (2021). Caracterización florística y estructural de los tipos de bosques por unidades fisiográficas en El Caimital, Barinas - Venezuela. Recursos Rurais, 17, 55-63. https://revistas.usc.gal/index.php/rr/article/view/7491

Rangel, Y. (2009). Caracterización ecopedológica del Sector " $b$ " del bosque universitario el caimital, municipio obispos, estado barinas, Venezuela [Tesis para obtener el título de ingeniero forestal, Universidad de Los Andes, Mérida - Venezuela].

Rosenzweig, M.L. (1995). Species diversity in space and time. Universidad de Cambridge.
Salazar, M., Vallejo, F., \& Salazar, F. (2019). Inventarios e índices de diversidad agrícola en fincas campesinas de dos municipios del Valle del Cauca, Colombia. Entramado, 15(2), 264-274. https://doi. org/10.18041/1900-3803/entramado.2.5744

Stiling, P. (1999). Ecology; theories and applications (3rd ed.). Prentice Hall.

Valdez, C., Guzmán, M., Valdés, A., Forougbakhch, R., Alvarado, M., \& Rocha, A. (2018). Estructura $\mathrm{y}$ diversidad de la vegetación en un matorral espinoso prístino de Tamaulipas, México. Revista de Biología Tropical, 66(4), 1674-1682. http://dx.doi. org/10.15517/rbt.v66i4.32135

Veillon, J. P. (1997). Los bosques naturales de Venezuela. Parte III. Los bosques tropófitos o veraneros de la zona de vida Bosque Seco Tropical. Universidad de Los Andes, Instituto Forestal Latinoamericano. https:// www.urbe.edu/UDWLibrary/InfoBook.do?id=5234

Vincent, L., Zambrano, T., \& Rodríguez, L. (2000). Manual de Inventario Dinámico con Base en Parcelas Permanentes en Bosque Tropical Alto. Ministerio del Ambiente y de los Recursos Naturales (M.A.R.N.). Dirección General Sectorial del Recurso Forestal. Organización Internacional para las Maderas Tropicales (OJ.M. T). Proyecto PO 49 I 94 REV. 1 (F). Establecimiento de una Red de Monitoreo Forestal Continuo (Parcelas Permanentes de Crecimiento) en las Reservas Forestales y Lotes Boscosos de Guayana.

Vincent, L. (1969). Estudio Sobre las Técnicas y la Factibilidad Económica de la Plantación en Lineas con Referencia al Bosque Experimental "El Caimital" Edo Barinas. Universidad de Los Andes.

Zarco-Espinosa, V.M., Valdez-Hernández; J.I., Ángeles-Pérez, G., \& Castillo-Acosta, O. (2010). Estructura y diversidad de la vegetación arbórea del parque estatal Agua Blanca, Macuspana, Tabasco. Universidad y Ciencia. Trópico Húmedo. 26(1), 1-17. http://www.scielo.org. $\mathrm{mx} /$ scielo.php?script=sci arttext\&pid=S0186-29792010000100001\&lng=es\&t $\operatorname{lng}=\mathrm{es}$

Zulle, F., Brassiolo, M., Gómez, C., \& Kees, S. (2015). Enriquecimiento forestal en fajas en un bosque explotado del Chaco Húmedo. Bosque, 36(2), 171-177. http://dx.doi.org/10.4067/S071792002015000200003 